\title{
Plug before pave in chronic aortic dissections?
}

\author{
Grayson H. Wheatley III, MD
}

\author{
From Siragusa Vascular, LLC, Nashville, Tenn. \\ Disclosures: Author serves as a consultant for Medtronic, Terumo Aortic, Endologix, and Ethicon. \\ Received for publication June 20, 2018; revisions received June 20, 2018; accepted for publication June 22, 2018; \\ available ahead of print Aug 7, 2018 \\ Address for reprints: Grayson H. Wheatley III, MD, 28 White Bridge Pike, Suite 200, Nashville, TN 37205 \\ (E-mail: ghw3rd@gmail.com). \\ J Thorac Cardiovasc Surg 2019;157:e69-70 \\ $0022-5223 / \$ 36.00$ \\ Copyright (C) 2018 by The American Association for Thoracic Surgery \\ https://doi.org/10.1016/j.jtcvs.2018.06.080
}

Endovascular repair of chronic type B aortic dissections (TBADs) remains a challenging clinical dilemma. The chronic dissection septum appears to be more resistant to the positive aortic remodeling effects associated with thoracic endovascular aortic repair (TEVAR) therapies than is seen with the endovascular repair of acute TBAD. As such, clinical outcomes of "paving" the true lumen of a chronic TBAD with a TEVAR approach and covering the proximal entry tear have inferior results relative to TEVAR for acute TBAD. ${ }^{1}$ In this issue of the Journal, Eleshra and colleagues ${ }^{2}$ share their approach for a novel catheter-based treatment of a patient with a chronic TBAD and associated aneurysm of the false lumen. ${ }^{2}$

The goals of TEVAR therapy for treatment of chronic TBAD with aneurysmal degeneration of the false lumen are as follows: (1) to cover the proximal entry tear with a TEVAR device, (2) to expand the true lumen actively with the TEVAR device, (3) to eliminate antegrade flow into the false lumen, and (4) to promote positive aortic remodeling along with complete thrombosis within the aneurysmal sac of the false lumen. However, retrograde flow into the false lumen through reentry fenestrations in the dissection septum distal to the TEVAR device, along with retrograde flow directly into the false lumen from intercostal and lumbar arteries, can compromise the success of the TEVAR procedure. Positive aortic remodeling fails in as many as $35 \%$ of patients with chronic TBAD treated with a TEVAR procedure as a result of persistent downstream flow into the false lumen from distal reentry tears. ${ }^{3}$

Several reports have described techniques of direct intervention into the false lumen associated with TEVAR (the "candy-plug" technique) as a way of facilitating complete thrombosis of the expanding false lumen. ${ }^{4}$ This adjunctive approach does not fully address the false lumen below the distal end of the TEVAR device, however, which can frequently be affected by numerous reentry tears (at the visceral branches or iliac arteries) and can maintain patency

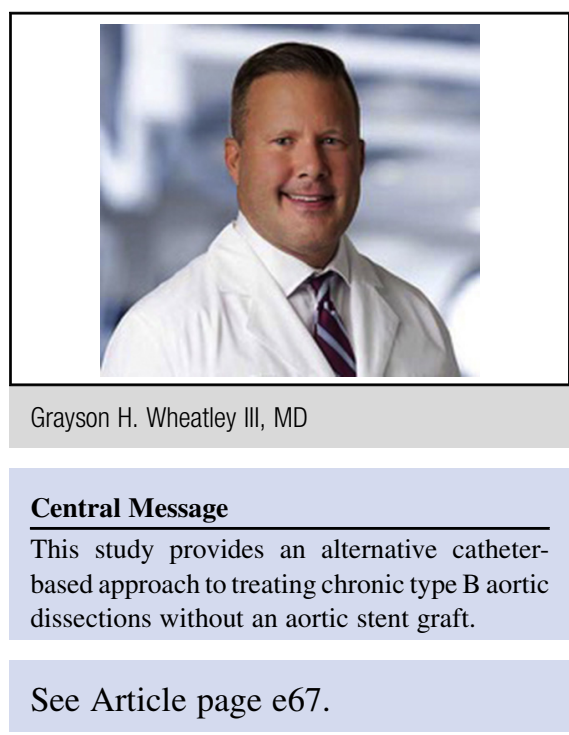

(as well as pressurization) of the false lumen in the visceral and abdominal aortic segments of the dissected aorta.

The novel approach ("stentless TEVAR") described in the report of Eleshra and colleagues ${ }^{2}$ takes a more comprehensive view of the TBAD. By using high-quality imaging, Eleshra and colleagues ${ }^{2}$ identified all of the entry and reentry tears in the dissection septum and developed a catheter-based approach to "plug" the sites of perfusion to the false lumen and add additional coils into the false lumen. The comprehensive, customized approach to the entire dissection septum (thoracic and abdominal) differs from TEVAR in the definition of treatment success. According to Eleshra and colleagues, ${ }^{2}$ the definition of treatment success is the elimination of all flow into the false lumen, with the goal of facilitating complete thrombosis of the entire false lumen. In theory, this approach would potentially decrease the number of reinterventions related to a patent visceral or abdominal false lumen and decrease late aortic-related deaths among patients with chronic TBAD. Furthermore, Eleshra and colleagues ${ }^{2}$ demonstrated that the diameter of the true lumen expands without the radial force of a TEVAR device. Additional patients need to be studied, and long-term follow-up is needed before we can recommend "plug" instead of "paving" for chronic TBAD.

\section{References}

1. Kang WC, Greenberg RK, Mastracci TM, Eagleton MJ, Hernandez AV, Pujara AC, et al. Endovascular repair of complicated chronic distal aortic dissections: intermediate outcomes and complications. J Thorac Cardiovasc Surg. 2011; $142: 1074-83$ 
2. Eleshra AS, Heo W, Lee K-H, Song S-W. Stentless thoracic endovascular aortic repair of a chronic DeBakey IIIb aneurysm. J Thorac Cardiovasc Surg. 2019; 157:e67-8.

3. Song SW, Kim TH, Lim SH, Lee KH, Yoo KJ, Cho BK. Prognostic factors for aorta remodeling after thoracic endovascular aortic repair of complicated chronic
DeBakey IIIb aneurysms. J Thorac Cardiovasc Surg. 2014;148:925-32. 933.e1; discussion 932-3.

4. Wu IH, Chan CY, Luo CM, Wang SS. Modified candy-plug device for aneurysmal false lumen occlusion in chronic type B aortic dissection. J Thorac Cardiovasc Surg. 2018;155:1970-2.e2. 